\title{
Design of an LCC current-output resonant converter for use as a constant current source
}

\author{
A. J. Gilbert, D. A. Stone, C. M. Bingham*, M. P. Foster \\ SHEFFIELD UNIVERSITY \\ Department of Electronic \& Electrical Engineering \\ Mappin Street, Sheffield, S1 3JD, U.K. \\ Tel.: +44/ (0)114 2225355 \\ *E-Mail: c.m.bingham@sheffield.ac.uk \\ URL: http://www.sheffield.ac.uk/eee
}

\section{Keywords}

Resonant converter, DC power supply, Soft switching, ZVS converters

\begin{abstract}
A methodology for the design of LCC resonant current-source converters, is presented. Unlike previous techniques, the resulting converter provides near constant steady-state output current over an extended load range when excited at the resonant frequency, through use of a self-oscillating controller.
\end{abstract}

\section{Introduction}

It is well established that resonant converters are advantageous in-terms of size and efficiency. This paper first establishes that an LCC current-output converter can be made to perform as a constant current-source at the resonant frequency and then provides a simple design process.

Many of the underlying equations used in the proposed methodology have been previously reported [1-3]. The design process utilises the accuracy of Fundamental Mode Approximation (FMA), at the resonant frequency, and the potential for rapid analysis that it provides. The analysis is based on the LCC current-output resonant converter shown in Figure.1.

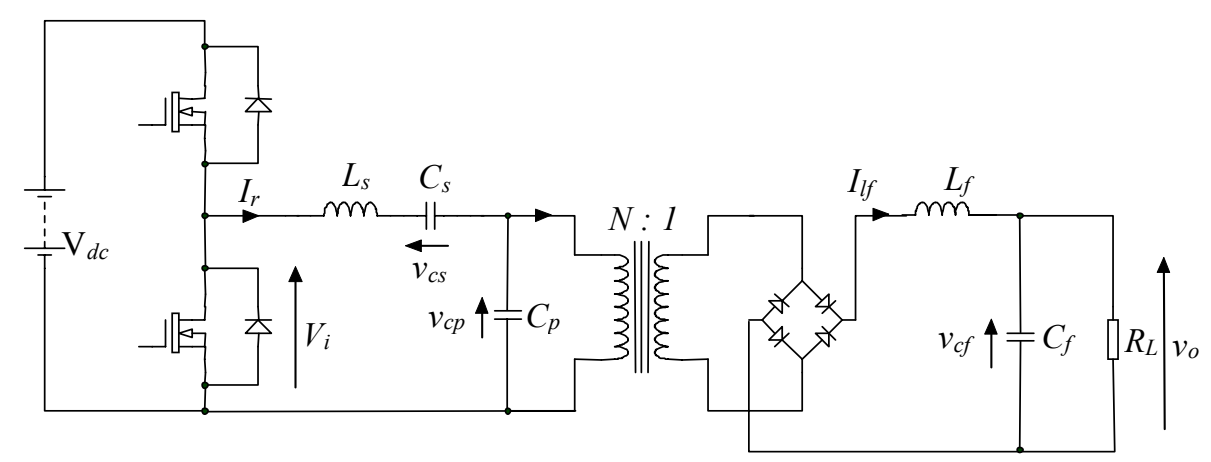

(a) 


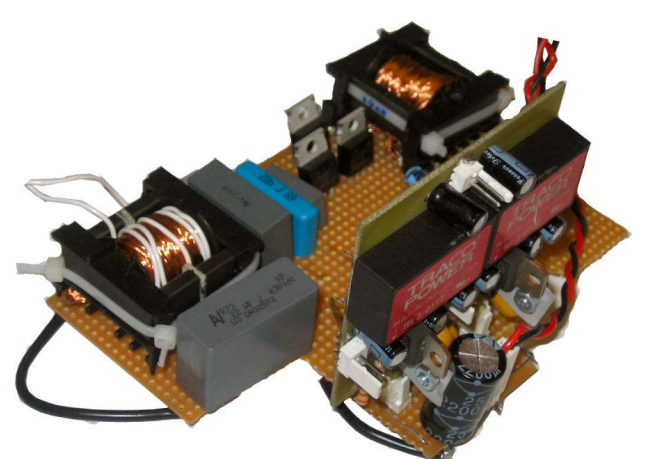

(b)

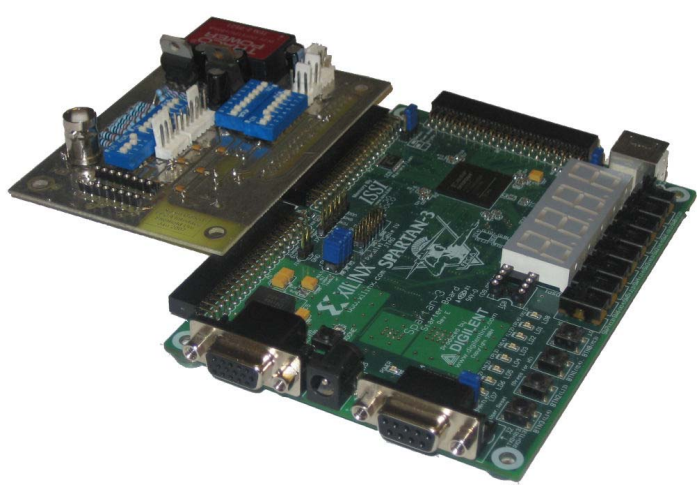

(c)

Fig. 1: LCC current-output resonant converter (a) schematic (b) prototype converter (c) self-oscillating phase lock controller

\section{Design Methodology}

In [3] it is shown that the tank components are related to the tank gain at resonance $G_{t r}$, the transformer turns ratio $N$, the resonant switching frequency $f_{r}$, the parallel to series capacitor ratio $\left(A=C_{p} / C_{s}\right)$ and load resistance $R_{L}$ as follows:

$C_{p}=\frac{\sqrt{G_{t r}^{2} \pi^{4}-16}}{\pi^{3} N^{2} R_{L} f_{r}}$

$L_{s}=\frac{N^{2} R_{L}\left((A+1) G_{t r}{ }^{2} \pi^{4}-16\right)}{4 \pi^{3} f_{r} G_{t r}{ }^{2} \sqrt{G_{t r}{ }^{2} \pi^{4}-16}}$

where $A=C_{p} / C_{s}, G_{t r}$ is the tank gain at resonance i.e $V_{o}=V_{i n} G_{t r} / N$ at resonance, $f_{r}$ is the resonant frequency and $R_{L}$ is the load resistance.

It is now shown that as the load resistance is increased, the converter output current tends to a constant value. Solving both (1) and (2) for $f_{r}$ equating the results and further solving for $G_{t r}$ gives:

$G_{t r}=\frac{\sqrt{2}}{4 \pi^{2} \sqrt{L_{s}}} \sqrt{64 L_{s}+N^{4} R_{L}^{2} C_{p} \pi^{4}(A+1)+X}$

where $X=\sqrt{4096 L_{s}{ }^{2}+128 L_{s} N^{4} R_{L}{ }^{2} C_{p} \pi^{4}(A-1)+N^{8} R_{L}{ }^{4} C_{p}{ }^{2} \pi^{8}(A+1)^{2}}$

Now, for a given output current, the tank gain at resonance can be calculated as follows:

$I_{o}=\frac{V_{i n} G_{t r}}{R_{L} N}$

substituting (3) into (4) gives an expression for the output current as load is varied and the converter switched at resonance. 
The output current ideally tends to a constant minimum value as the load resistance is increased and the converter switched at resonance. This can be shown by taking the limit of (4) as $R_{L} \rightarrow \infty$ (opencircuit) after $G_{t r}$ is eliminated through the use of (3) giving:

$$
I_{o_{-} \min }=\frac{V_{i n} N \sqrt{C_{p}} \sqrt{A+1}}{2 \sqrt{L_{s}}}
$$

This is the minimum output current the converter will produce when excited about the resonant frequency. When the load resistance is lowered, the output current will be larger. Given that the maximum current (at minimum load resistance) is $K$ times larger than the minimum current $\left(I_{o_{-} \max }=K . I_{o_{-} \min }\right)$ allows the required value of $A$ to be calculated. Solving (5) for $C_{p} / L_{s}$ then yields:

$$
\frac{C_{p}}{L_{s}}=\frac{4 I_{o_{-} \min }^{2}}{N^{2}(A+1) V_{\text {in }}^{2}}
$$

Dividing (1) by (2), eliminating $G_{t r}$ through the use of (4), replacing $I_{o}$ with $K . I_{o_{-} \text {min }}$ and $R_{L}$ with $R_{L_{-} \text {min }}$, equating the result with (6) and finally solving for $A$ gives:

$$
A=-\frac{I_{o_{-} \min }{ }^{2} N^{2} R_{L_{-} \min }{ }^{2} \pi^{4} K^{2}\left(K^{2}-1\right)+16 V_{\text {in }}{ }^{2}\left(1-K^{2}\right)}{K^{2}\left(I_{o_{-} \min }{ }^{2} N^{2} R_{L_{-} \min }{ }^{2} \pi^{4}\left(K^{2}-1\right)-16 V_{\text {in }}{ }^{2}\right)}
$$

It is now possible to generate a constraint on the minimum and maximum allowed values of $N$ for a given design specification. To generate the minimum, the value of $A$ must never be -ve, hence, solving for $N$ in (7) at $A=0$ gives:

$$
\frac{4 V_{\text {in }}}{I_{o_{-} \min } R_{L_{-} \min } K \pi^{2}}<N
$$

To generate the maximum value, solving (7) for the limit of $N$ as $A \rightarrow \infty$ gives the following constraint:

$$
N<\frac{4 V_{\text {in }}}{\pi^{2} R_{L_{-} \min } I_{o_{-} \min } \sqrt{K^{2}-1}}
$$

Hence, for a given specification, the range of $N$ must first be calculated, the transformer turns ratio $N$ specified, the capacitor ratio $A$ calculated from (7), the $G_{t r}$ calculated at the minimum load by assuming $I_{o}=K . I_{o_{-} \min }$ and solving (4), and finally computing the required components from (1) and (2). The minimum resonant frequency corresponds to $f_{r}$. The peak resonant frequency will correspond to the resonant frequency of the converter with the rectifier disconnected.

The methodology is now applied to the design of a constant current converter. The specifications for the supply are provided in Table 1.

Table 1 - DC-DC Converter Specifications

\begin{tabular}{|l|l|l|}
\hline $\begin{array}{l}\text { DC Input } \\
\text { Voltage }\end{array}$ & $\begin{array}{l}\text { Current source } \\
\text { Range }\end{array}$ & Output voltage Range \\
\hline $18 \mathrm{~V}$ & $0 \rightarrow 1 \mathrm{~A}$ & $10 \rightarrow 20 \mathrm{~V}$ \\
\hline
\end{tabular}

The theoretical open circuit output current, at resonance, is selected to be $10 \%$ larger than the required peak current, thereby ensuring that parasitic effects (such as diode voltage drops) will not reduce the 
current so as to make the design ineffective and will ensure above-resonance operation at the peak required output current,. Therefore $I_{o_{-} \text {min }}=1.1 \mathrm{~A}$. Now, selecting $K=1.1$ ensures that at the minimum specified load resistance, the output current at resonance will not exceed 1.21A. Equations (8) and (9) provide a constraint on the range of $N$. At 1 A output, the minimum load resistance is $10 \Omega$, hence $0.60<\mathrm{N}<1.45$. Selecting an $N$ of 1 for simplicity and reduction in converter cost, gives a $G_{t r}=0.672$ and from (7) the value of $A$ is therefore found to be 0.582. Assuming a minimum switching resonant frequency of $133 \mathrm{kHz}$, the required tank components from (1) and (2) are $C_{p}=128 \mathrm{nF} C_{s}=220 \mathrm{nF}$ and $L_{s}=13.6 \mathrm{uH}$.

For the component values selected above, Fig. 2 provides the steady-state output current when switched at resonance, as the load is varied. The data is obtained from a prototype converter, ideal simulation, and design process (see equations (3) \& (4)).

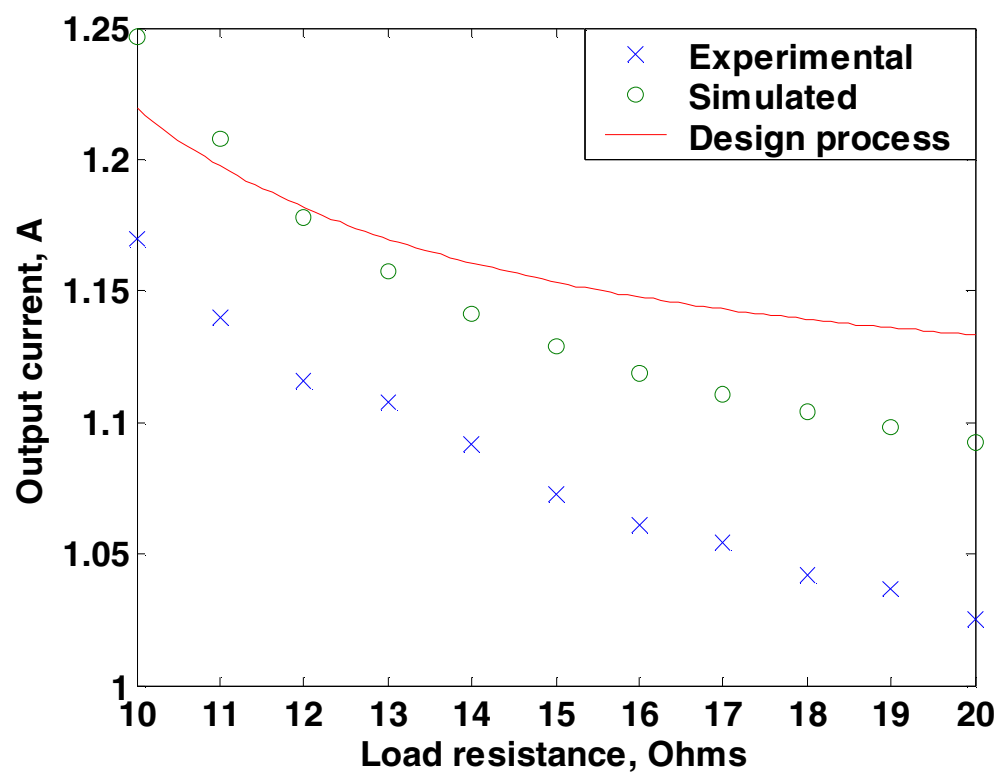

(a)

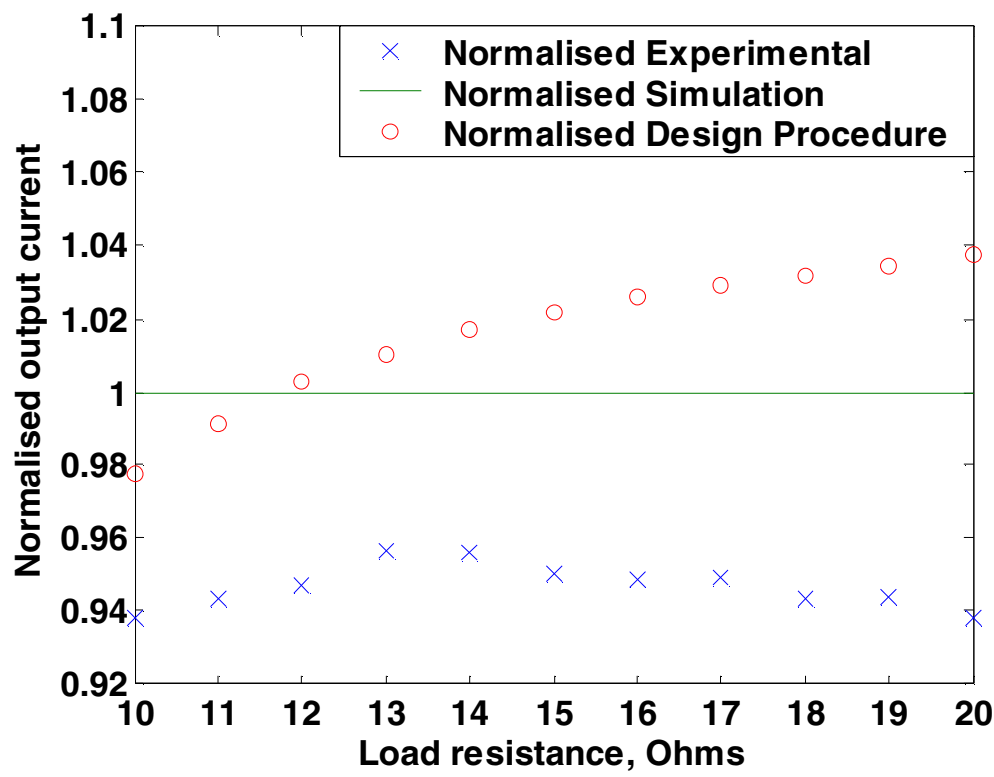

(b)

Fig. 2: (a) Output current (b) normalised output current w.r.t ideal converter simulation as load is varied from $5 \Omega$ to $10 \Omega$ when switched at resonance 
From Fig. 2(a) it can be seen that the prototypes' steady-state output current varies little as the load is changed and the converter switched at resonance. Since a series-inductor resistance of $0.15 \Omega$ is present in the converter, it is expected that the experimental output-current data will be slightly lower than that of the ideal simulated converter. Note that from an ideal perspective the presence of a forward voltage drop should not reduce the output current below the theoretical open-circuit current since the diodes effectively make the load resistance appear larger, thus forcing the converter to naturally compensate by increasing the resonant tank current. Figure 2(b) provides a measure of error in the proposed analysis. In the presented data, the largest error between the analysis and simulated ideal converter is approximately $4 \%$. The maximum error between experimental output-current and the analysis occurs at the highest output power, and is approximately $10 \%$. This makes the design process ideal for prototype component selection especially when one considers the simplicity of the equations.

A benefit of the proposed design procedure, when a constant load current is desired, is the ability to operate near resonance across the full load range. This maximises the converter efficiency since less reactive power is present in the resonant tank. Figure 3, provides the efficiency of the prototype converter for the load range specified in Table 1, when switched at resonance.

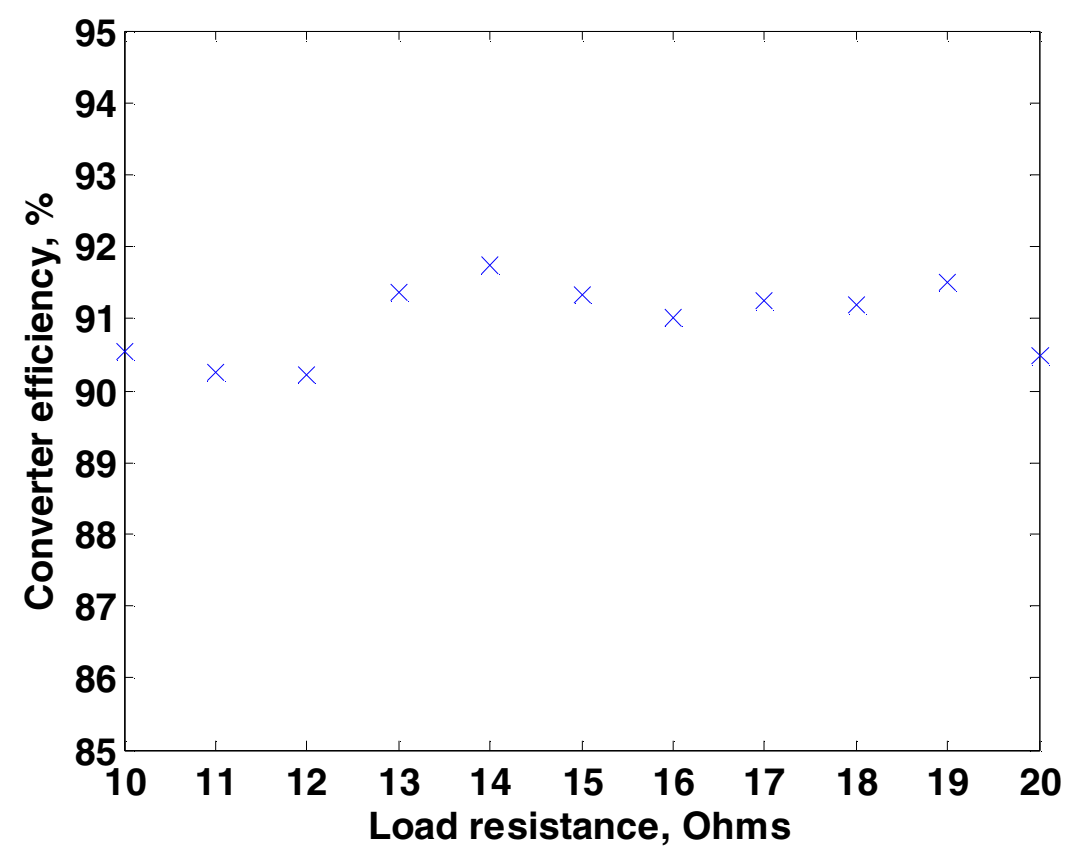

Fig. 3: Prototypes efficiency when operated at resonance over the specified load range

\section{Conclusion}

An LCC resonant converter current-source design methodology, based on the FMA analysis technique, has been presented. The steady-state output current has been shown to remain near constant over an extended load range when switched at the resonant frequency. If supplied by a constant voltage, the converter is capable of virtually constant output-current regulation in open-loop via a selfoscillating at resonance switching mechanism. Results from a prototype converter have validated the proposed methodology. 


\section{References}

[1] R. L. Steigerwald, "A comparison of Half-Bridge Resonant Converter Topologies," IEEE Trans. Power Electronics, vol.3, no.2, April 1988, pp.174-182.

[2] Marian K. Kazimierczuk, Dariusz Czarkowski, Resonant Power Converters, John Wiley sons, inc 1995, ISBN 0-471-04706-6, Chapter 8 \& 17.

[3] A. J. Gilbert, D. A. Stone, C. M. Bingham, "Rapid design of LCC current-output resonant converters with reduced electrical stresses", Power Electronics and Applications, 2005 European conference on 11-14 Sept 2005. 